\title{
Generating Timed Artificial Dream Action(TADA) as the Inverse Process of Freud's Interpretation of Dreams
}

\author{
M. A. El-dosuky \\ Department of Computer Sciences, Faculty of Computer and Info., Mansoura University, \\ P.O. 35516, Egypt
}

\begin{abstract}
Apart from being an audacious attempt and a masterpiece, Freud's Interpretation of Dreams has provoked controversy since its publication. After the neurological linkage between Rapid Eye Movement(REM) sleep and dreams, many hypotheses are proposed focusing on REM or its accompanied dreams. This paper reviews recent discoveries regarding the neuro-cognitive aspects of sleep, dreaming, and emotions as a dream ingredient. This paper assumes that a dream content is decomposable into a sequence of Timed Artificial Dream Actions (TADAs) and that dream interpretation resembles the ability of an expert system explaining HOW and WHY questions. Freud tried to answer WHY each dream ingredient is being incorporated. The inverse of this process is to answer HOW a TADA is formed, and it is the concern of this paper. Based on dream-contents, an operational model for dream ingredients is proposed. The proposed TADA generator, nicknamed Oneiros, is decomposed into three modules Morpheus, Phantasos and Phobetor. Morpheus is responsible for the lexical processing of memory contents, in order to perform tasks such as extracting objects, emotions associated with objects, and any object alterations. Phobetor is responsible for extracting viewer emotions, and phobetic-specific aspects. Phantasos is responsible for the actual generation of a TADA.
\end{abstract}

Keywords: Rapid Eye Movement(REM) sleep, Dream Action, NGram

\section{Introduction}

Dreams have been bewildering humanity, initiating endless hypotheses about their source, meaning and function (Hobson (2009)). 
Apart from being a masterpiece, Freud (1900) has provoked controversy since its publication, oppugning literal usage of theories advanced within. In this audacious attempt, Freud argued that a dream, as a wish-fulfilment, has a symbolic meaning. Freud's analysis of a dream of his own, a "Dream of July 23-24, 1895", exemplifies his method of interpretation on many dreams. The dream starts with "A great hall - a number of guests, whom we are receiving - among them Irma...", and henceforth it shall be referred to as the "Dream about Irma". In his analysis of this aforementioned dream, Freud decompose it into clauses.

This paper is based on a couple assumptions. The first of these core assumptions, is that a dream is decomposable into a sequence of Timed Artificial Dream Actions (TADAs). The basic unit of a dream is a dream action, such as seeing oneself flying, or seeing someone dying. Dream actions are to a dream as clauses are to story. Freud himself decompose his dream in order to analyze it, as in the aforementioned dream about Irma. Timing and artificiality are two aspects of a dream action that need to be insisted here. A dream action is timed, in the sense that it has sequence ID as a scene in a movie reel. It is artificial, in the sense that the dreamer, once is awake, can distinguish it from awake actions.

The second of core assumptions, is that dream interpretation resembles the explanation facility in an expert system (ES). Explanation, a.k.a. justification, denotes the ability of an ES to explain its behavior (Merritt (2001)). The declarative reading of an ES as a logic program enables the explanation of HOW or WHY questions (Denecker (2000)), by working backwards or forwards respectively. In one hand, to answer HOW a conclusion is reached, work backwards. In the other hand, to answer WHY a computation is being performed, the ES works forwards to its current goal. Following this analogy, we can assume that certain memory ingredients contribute to the formation of a TADA, as shown in Figure 1, in which the TADA is formed due of ingredients $1,2, \ldots n$. To answer HOW a TADA is formed, work backwards. To answer WHY an ingredient is being recalled from memory, work forwards to the current TADA. Contemplating Freud's attempt, it turns out that he tried to answer WHY each ingredient is being fetched from memory. The inverse of this process is to answer HOW a TADA is formed, and it is the concern of this paper.

The rest of the paper is organized as follows. Section 2 is a background that reviews literature on sleep in subsection 2.1, dreaming in subsection 2.2, and emotions as a dream ingredient in subsection 2.3 . 


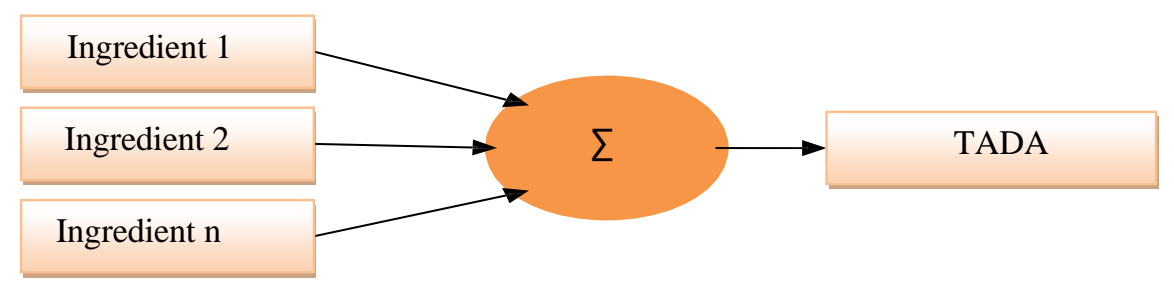

Figure 1: TADA

Section 3 presents the proposed system for generating Timed Artificial Dream Action(TADA).

Sections 4 and 5 are for conclusion and future work, respectively.

\section{Background}

\subsection{Sleep}

Many experiments investigate the role and the function of sleep in brain. Sleep notably orchestrates the metabolite clearance (Xie et al. (2013) $)$, consolidates memory (Marshall et al. (2006), Mehta (2007)), contributes to learning(Gais et al. (2002), Stickgold et al. (2001)), and inspires insight(Wagner et al. (2004)).

\subsection{Dreaming}

For a recent survey for theories on the function of dreaming, you can refer to Sayed (2011) which groups them into

1. Psycho-dynamic such as Freud (1900), Jung (1967), and Solms (1997).

2. Evolutionary such as Revonsuo (2000), Franklin \& Zyphur (2005) and Barrett (2007)

3. Neuro-cognitive such as Domhoff (2001), Hobson \& McCarley (1977), Stickgold et al. (2001), Stickgold et al. (2000), Louie \& Wilson (2001) and Maquet et al. (2002).

After the neurological linkage between Rapid Eye Movement(REM) sleep and dreams (Snyder (1966)), many hypotheses are proposed focusing on REM or accompanied dreams. The major trend is the memory consolidation hypothesis(Stickgold et al. (2000), Louie \& Wilson (2001), Maquet et al. (2002)). 
Recent studies impugn this trend based on the occurrence of consolidation in non-REM sleep. Hobson (2009), for instance, proposes the 'protoconscious hypothesis' in which REM sleep contributes in constituting a state, that helps in developing and maintaining the waking consciousness afterwards. An interesting aspect about this hypothesis, is that it nods to a REM world model based on computer games or virtual reality.

However, Klemm (2011) argues that this hypothesis is not testable, and proposes that, given enough sleep, REM is used by a brain to enable it in waking-up, hence the name 'wake-up hypothesis'. Based on this view, brain consolidates memories as a by-product of its continual operation in sleep on available information. Klemm (2011) attempts to make his hypothesis stands out among competing hypotheses, by grounding it upon many supporting facts that he provided. He agrees with Vertes (1986) who proposed 'life-sustaining hypothesis' that REM is a mechanism that supplies the brain with endogenic simulations to forbid it from being excessively shutdown in Slow-wave sleep (SWS). Klemm (2011) assails the hypothesis proposed by Hobson \& McCarley (1977) on the randomness of REM neuronal firing, causing stories, a.k.a dreams, to be generated in order to rationalize any erratic action.

\subsection{Emotions}

The relation between emotions and cognition is undeniable(Pessoa (2008)). From evolutionary perspective (Plutchik (1980)), emotions contribute to survival (Grof (1988)). Fear is contributed to amygdala (Davis (1992)), isn't it?(LeDoux (2015)).

Ekman (1992) attempts to spicily basic emotions, defined as discrete responses to universally shared events (Ekman \& Cordaro (2011)). Contempt, for instance, is proven to be basic (Ekman \& Heider (1988)).

\section{Proposed System}

Based on dream-contents, let us propose an operational model for dream ingredients as shown in figure 2 .

The Emotions set adopts the basic emotions of neutral (abbreviated as $\mathrm{n}$ ), contempt (abbreviated as c), happy (abbreviated as h), surprise (abbreviated as s), grief (abbreviated as g), disgust (abbreviated as d), fear (abbreviated as f) and anger (abbreviated as a). Note that, here, sadness is called grief 


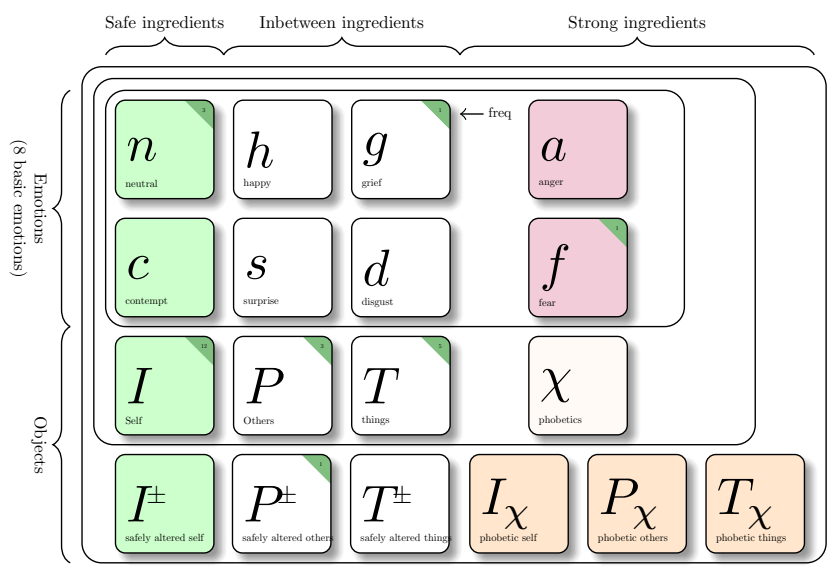

Figure 2: Operational dream ingredients

just to distinguish it from surprise when abbreviated. In context of emotions as a dream ingredients, it is worth contrasting the two basic emotions of contempt and fear. Unpleasant dreams, a.k.a, nightmares, usually have strong emotional brain responses typically fear, indicating a sense of danger. On contrary, contempt indicates superiority. It is said that familiarity breeds contempt. That is why I feel urged to consider emotionally classified ingredients as contempt to be safe.

The Objects set encompasses the dream-viewer (abbreviated as I), other people (abbreviated as $\mathrm{P}$ ) and all things both animate and inanimate (abbreviated as T). The dichotomy of objects, other than the dream-viewer, into $\mathrm{P}$ and $\mathrm{T}$ reflects the difference between them in terms of possible interactions with the viewer.

Safely altered occurrences of the dream-viewer (abbreviated as $I^{ \pm}$), of other people (abbreviated as $P^{ \pm}$) and of things (abbreviated as $T^{ \pm}$).

Phobetics, denoted as $\chi$, are fearful objects, such as Vampire, werewolf, and the like. A recent study scrutinize the conceptualization of BeastPeople(Pizzato (2016)). In context of emotions as a dream ingredients, I may assume that a phobetic, per se, does not cause fear, unless attached to a fear ingredient. Phobetic occurrences of the dream-viewer (abbreviated as $I_{\chi}$ ), of other people (abbreviated as $P_{\chi}$ ) and of things (abbreviated as $T_{\chi}$ ).

The freq of an ingredient is shown at its upper right corner, signifying the total number of occupance in the dream content based on a rough estimation. 


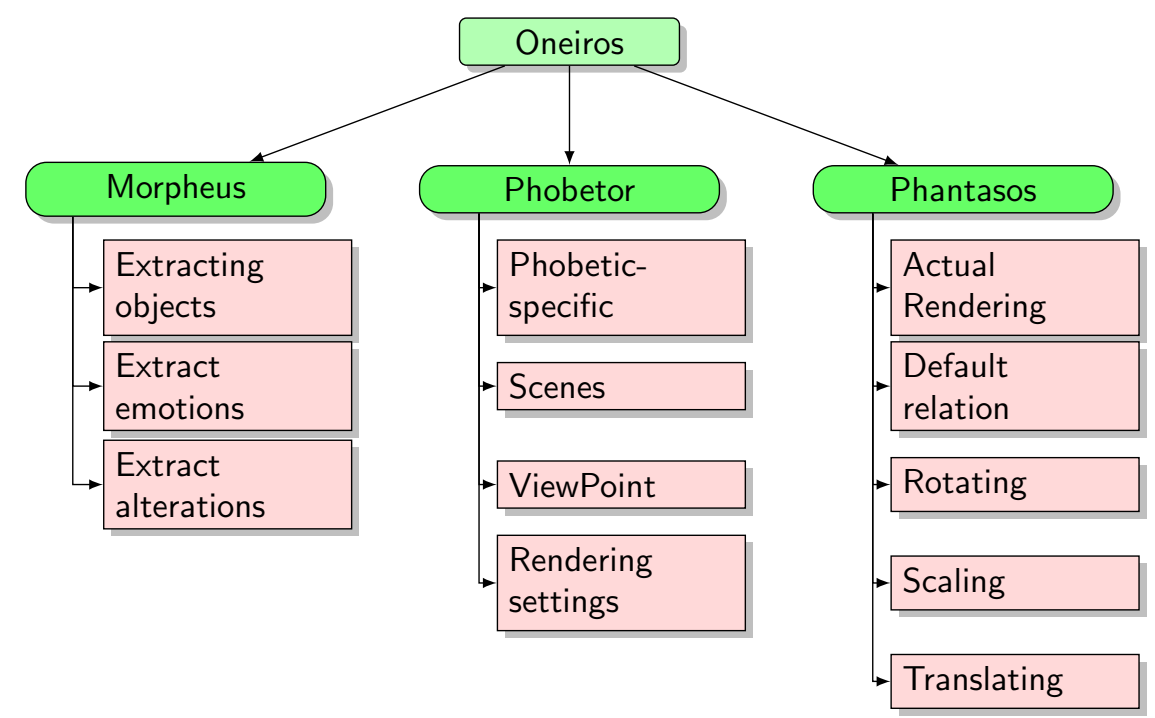

Figure 3: Oneiros modules

The proposed system, nicknamed Oneiros, is decomposed into three modules Morpheus, Phantasos and Phobetor, based on names from Greek and Roman Mythology (Smith (1849)) relating to dream.

\subsection{Morpheus}

This module is responsible for the lexical processing of memory contents, in order to perform tasks such as extracting objects, emotions associated with objects, and any object alterations. To clarify these tasks, let us provide Python code snippets to one of theses tasks. You need to download and install Python. For a matter for computability, please download any version beneath version 2.9, 2.6 is OK. Then obtain and install the following packages

- PyYAML

- NLTK

- Numpy

- Matplotlib 
Extract the text of any file to denote the memory content till now. For a matter of simplicity, the "Dream about Irma" is fetched and put in a file named 'memoryContent.txt'. Note that this content is a dream for Freud, but for us it is just a memory as we read it. Open the installed Python (IDLE), and write the following code.

$\mathrm{f}=\operatorname{open}($ 'C: $\backslash \backslash$ memoryContent.txt' $)$

raw $=\mathrm{f} \cdot \operatorname{read}()$

This code reads the contents of the file. Then import the NLTK library (Bird (2006)), and tokenize the read data.

import nltk

tokens $=$ nltk. word_tokenize $($ raw $)$

Then let us convert the tokens into a Text data type, which allows for extensive textual operations.

memory $1=$ nltk. Text $($ tokens $)$

For example, it provide the collocations() function.

memory1.collocations ()

Calling it gives the following result: friend Otto, meaning that these two words co-occur together.

Now its time to extract ingredients,

freqDist $1=$ memory 1 . vocab ()

This code calculate the frequency distribution of words in memory content into a variable named freqDist1 of FreqDist data type. This data type allows for many useful operations such as freqDist1.tabulate() to tabulate the FreqDist, freqDist1.max () to get the most frequent word, freqDist1.hapaxes() to get the least frequent words that only appear once.

To trace the appearance of key characters in the memory content, we can use Dispersion Plot as shown in Figure 4 for 'I','Irma' and 'Otto' words.

memory1. dispersion_plot (['I ', 'Irma', 'Otto']) 


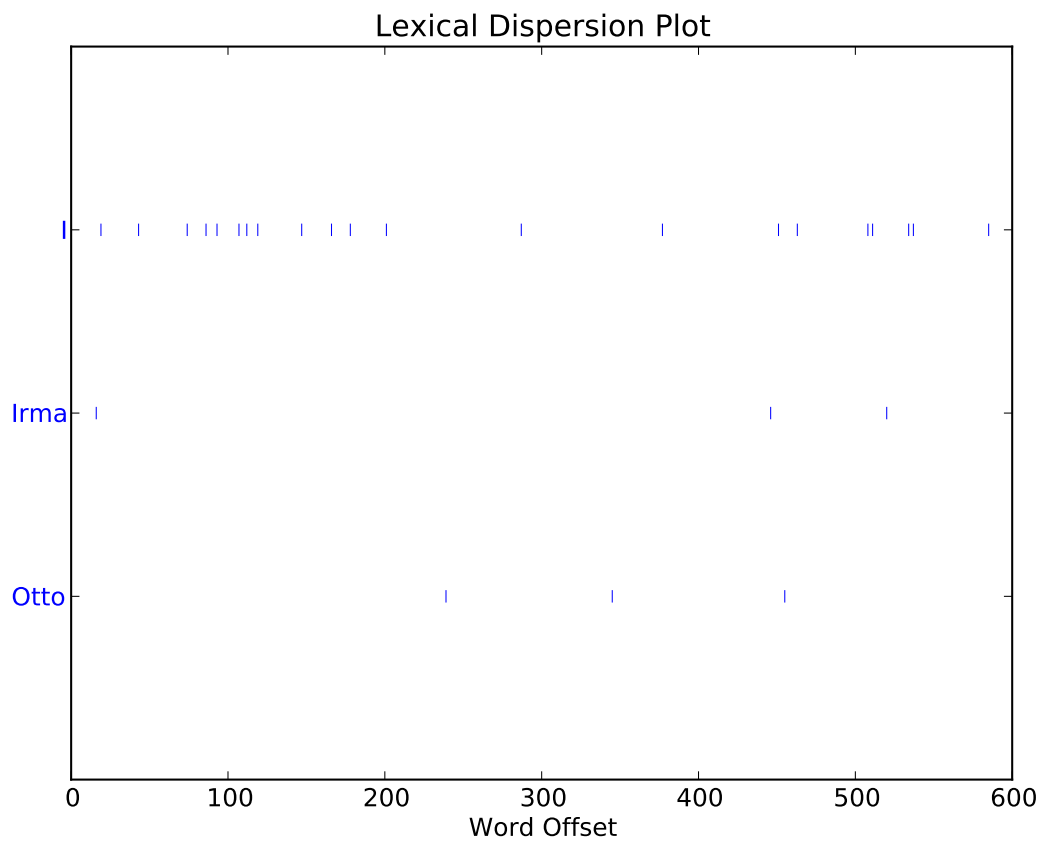

Figure 4: Dispersion plot for I, Irma and Otto 


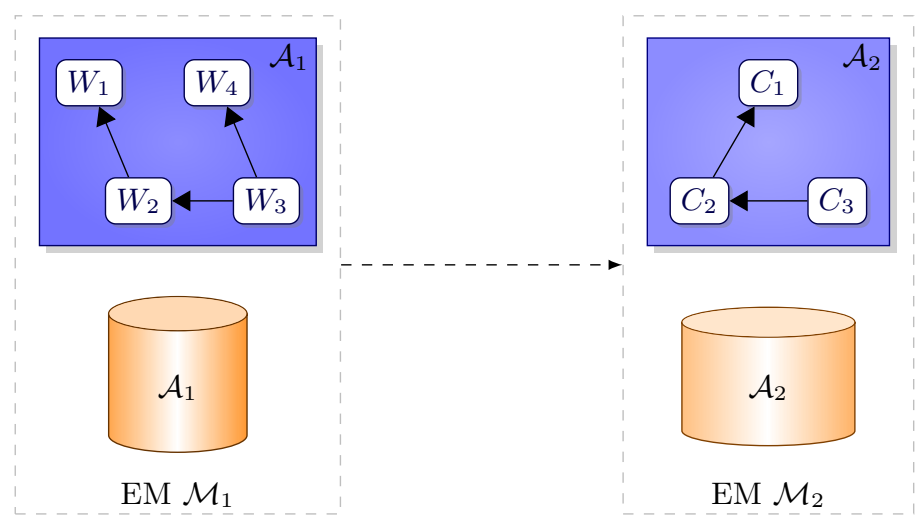

Figure 5: Generating a TADA using a specific pattern

\subsection{Phobetor}

The main task of the Phobetor module is being responsible for extracting viewer emotions, and all phobetic-specific aspects all phobetic-specific aspects. Viewer emotions are totaly different from emotion associated with objects. The former is a form of a attitude or a sentiment that makes it difficult to infer, compared to the later which is easier to extract. "The smiling vampire is approaching me", for instance, may easily associate happiness to the vampire object. But the viewer may feel frightened. Minor Phobetor tasks include determining the parameters for scenes, camera, and rendering settings. However, this version is limited to textual output.

\subsection{Phantasos}

This module is responsible for the actual generation and rendering of a TADA. In a textual output, the Default relation is assumed. However, in visual output, there could be possible Rotating, Scaling and Translating of objects.

The TADA can be generated using specific patterns, as in ELIZA (Weizenbaum (1966)) as shown in Figure 5. So the Action $A_{1}$ in Episodic Memory $M_{1}$ is used as a template in generating the Action $A_{2}$ in Episodic Memory $M_{2}$. But, I rather prefer generating TADAs using n-gram model (Lin \& Hovy (2003)) which is used in text summarization.

memory1. generate () 
All TADAs generated form "Dream about Irma", start with the sentence "A great hall - a number of guests,". The rest of 7 arbitrarily generated textual TADAs are as follows :

- "whom I immediately take aside, as though to answer her letter...",

- "whom we are receiving - among them Irma, whom I immediately take aside, as though to answer her letter..."

- "whom I immediately take aside , as though to answer her letter..."

- "whom we are receiving - among them Irma, whom I immediately take aside, as though to answer her letter..."

- "whom we are receiving - among them Irma, whom we are receiving - among them Irma, whom I immediately take aside, as though to answer her letter..."

- "whom we are receiving - among them Irma, whom I immediately take aside, as though to answer her letter..."

- "whom I immediately take aside, as though to answer her letter..."

A final note is to consider the interaction between these three modules, as shown in Figure 6 The Self can be operationally approximated by the profile of the dreamer containing his or her characteristics and mental image. First, Oneiros initializes operation. Morpheus identifies ingredients. Then, Phobetor evaluates them for determining the existence of strong emotions. If the candidate-TADA is safe to be germinated, Phantasos generates it. Otherwise there is a great probability to cause arousal wake-up.

\section{Conclusion}

Freud tried to answer WHY each dream ingredient is being incorporated. The inverse of this process is to answer HOW a TADA is formed, and it is the concern of this paper. Based on dream-contents, an operational model for dream ingredients is proposed. The proposed TADA generator, nicknamed Oneiros, is decomposed into three modules Morpheus, Phantasos and Phobetor. Morpheus is responsible for the lexical processing of memory contents, in order to perform tasks such as extracting objects, emotions associated with 


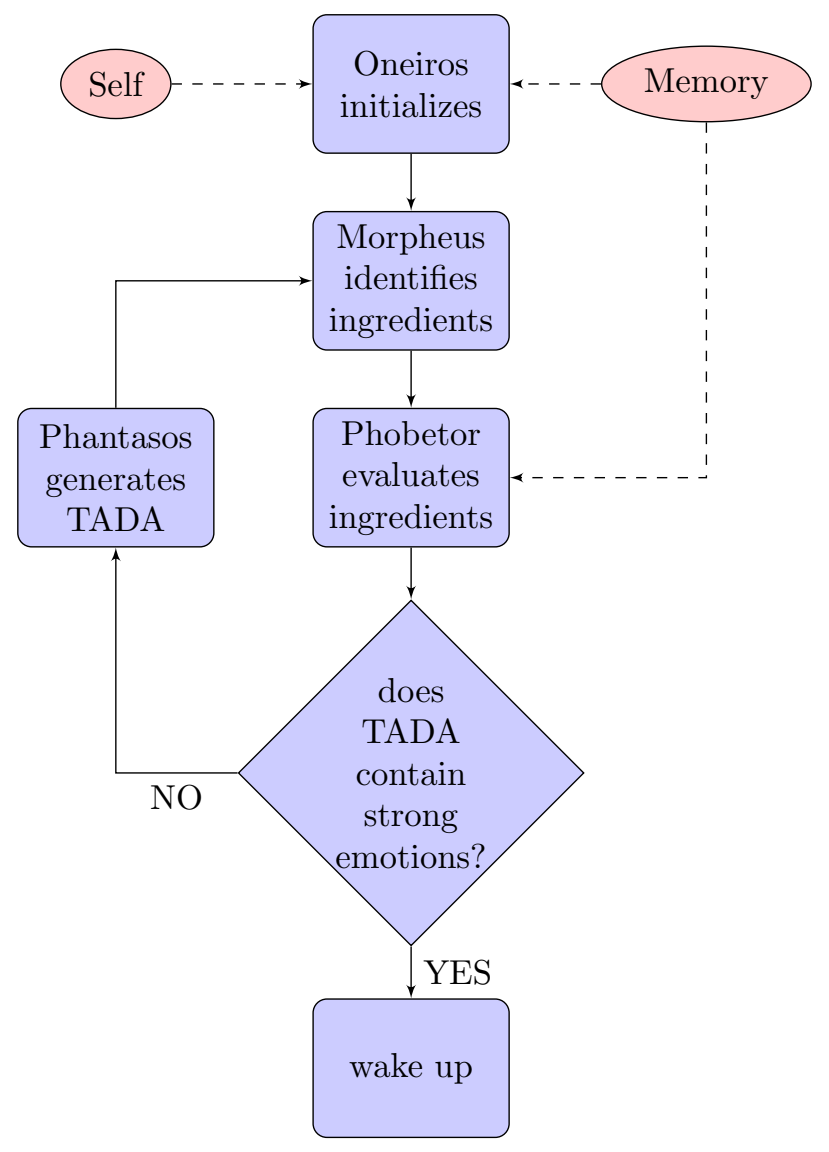

Figure 6: flowchart 
objects, and any object alterations. Phobetor is responsible for extracting viewer emotions, and all phobetic-specific aspects. Phantasos is responsible for the actual generation of a TADA.

If the N-Gram perfectly approximates the memory consolidation, then we can end up with a couple interesting observations :

- All TADAs generated form "Dream about Irma", start with a sentence from the starting part of the memory content, which corresponds to earliest impressions of childhood.

- some of the arbitrarily generated TADAs contains recursive patterns such as "among them Irma, whom I ... - among them Irma , whom I..."

\section{Future Work}

Frequency distribution of ingredients of memory content is based on a rough estimation, Thus, "I" and "me", for instance, and "Irma" and "she" (refereeing to Irma) do not add up. This may require a refinement based on comprehension of the memory content. Also, building the semantic knowledge from memory content can be based on the application of WordNet (Miller (1995)) and/or ConceptNet (Liu \& Singh (2004), Speer et al. (2017))

One possible direction is implementing visual output in Phobetor module, by determining the parameters for scenes, camera, and rendering settings. This may utilize any a virtual reality or game engines.

Another possible direction is to apply the proposed system in HumanRobot Interaction (El-dosuky (2014) $)$, by allowing a robot to dream and tell the generated dream to a human.

\section{References}

Barrett, D. (2007). An evolutionary theory of dreams and problem-solving. The New Science of Dreaming: Content, Recall and Personality Correlates, (pp. 133-154).

Bird, S. (2006). Nltk: the natural language toolkit. In Proceedings of the COLING/ACL on Interactive presentation sessions (pp. 69-72). Association for Computational Linguistics. 
Davis, M. (1992). The role of the amygdala in fear-potentiated startle: implications for animal models of anxiety. Trends in pharmacological sciences, $13,35-41$.

Denecker, M. (2000). A note on the declarative reading (s) of logic programming. arXiv preprint cs/0003056, .

Domhoff, G. W. (2001). A new neurocognitive theory of dreams. Dreaming, $11,13-33$.

Ekman, P. (1992). An argument for basic emotions. Cognition $\&$ emotion, $6,169-200$.

Ekman, P., \& Cordaro, D. (2011). What is meant by calling emotions basic. Emotion Review, 3, 364-370.

Ekman, P., \& Heider, K. G. (1988). The universality of a contempt expression: A replication. Motivation and emotion, 12, 303-308.

El-dosuky, M. A. (2014). HKH : Helen keller heuristic, a common ground scenario for human-robot interaction. International Journal of Computer Applications, 89, 1-6.

Franklin, M. S., \& Zyphur, M. J. (2005). The role of dreams in the evolution of the human mind. Evolutionary Psychology, 3.

Freud, S. (1900). The Interpretation of Dreams (Joyce Crick, Trans). Oxford University Press.

Gais, S., Mölle, M., Helms, K., \& Born, J. (2002). Learning-dependent increases in sleep spindle density. The Journal of neuroscience, 22, 68306834.

Grof, S. (1988). Modern consciousness research and human survival. Human survival and consciousness evolution, (pp. 57-79).

Hobson, J. A. (2009). Rem sleep and dreaming: towards a theory of protoconsciousness. Nat Rev Neurosci, 10, 803-813.

Hobson, J. A., \& McCarley, R. W. (1977). The brain as a dream state generator: an activation-synthesis hypothesis of the dream process. Am J Psychiatry, 134, 1335-1348. 
Jung, C. G. (1967). Collected Works of CG Jung, Volume 8 volume 8. Princeton University Press.

Klemm, W. (2011). Why does rem sleep occur? a wake-up hypothesis. Frontiers in systems neuroscience, 5.

LeDoux, J. (2015). The Amygdala Is NOT the Brain's Fear Center.

Lin, C.-Y., \& Hovy, E. (2003). Automatic evaluation of summaries using ngram co-occurrence statistics. In Proceedings of the 2003 Conference of the North American Chapter of the Association for Computational Linguistics on Human Language Technology-Volume 1 (pp. 71-78). Association for Computational Linguistics.

Liu, H., \& Singh, P. (2004). ConceptNet: A lexical database for english. BT Technology Journal, 4, 211-226.

Louie, K., \& Wilson, M. A. (2001). Temporally structured replay of awake hippocampal ensemble activity during rapid eye movement sleep. Neuron, 29, 145-156.

Maquet, P., Peigneux, P., Laureys, S., \& Smith, C. (2002). Be caught napping: you're doing more than resting your eyes. Nature Neuroscience, 5, 618-619.

Marshall, L., Helgadóttir, H., Mölle, M., \& Born, J. (2006). Boosting slow oscillations during sleep potentiates memory. Nature, 444, 610-613.

Mehta, M. R. (2007). Cortico-hippocampal interaction during up-down states and memory consolidation. Nature neuroscience, 10, 13-15.

Merritt, D. (2001). Building expert systems in prolog. Amazi. inc, 5861.

Miller, G. (1995). Wordnet: A lexical database for english. Communications of the ACM, 11, 39-41.

Pessoa, L. (2008). On the relationship between emotion and cognition. Nature Reviews Neuroscience, 9, 148-158.

Pizzato, M. (2016). Beast-People Onscreen and in Your Brain: The Evolution of Animal-Humans from Prehistoric Cave Art to Modern Movies. ABCCLIO. 
Plutchik, R. (1980). Emotion: A psychoevolutionary synthesis. Harpercollins College Division.

Revonsuo, A. (2000). The reinterpretation of dreams: An evolutionary hypothesis of the function of dreaming. Behavioral and Brain Sciences, 23, 877-901.

Sayed, L. (2011). The function of dreams and dreaming: moving towards an integrated understanding. Master's thesis.

Smith, W. (1849). Dictionary of Greek and Roman Biography and Mythology.

Snyder, F. (1966). Toward an evolutionary theory of dreaming. American Journal of Psychiatry, 123, 121-136.

Solms, M. (1997). The neuropsychology of dreams: A clinico-anatomical study. L. Erlbaum.

Speer, R., Chin, J., \& Havasi, C. (2017). Conceptnet 5.5: An open multilingual graph of general knowledge. In $A A A I$.

Stickgold, R., Hobson, J. A., Fosse, R., \& Fosse, M. (2001). Sleep, learning, and dreams: off-line memory reprocessing. Science, 294, 1052-1057.

Stickgold, R., Malia, A., Maguire, D., Roddenberry, D., \& O'Connor, M. (2000). Replaying the game: Hypnagogic images in normals and amnesics. Science, 290, 350-353.

Vertes, R. P. (1986). A life-sustaining function for rem sleep: A theory. Neuroscience and Biobehavioral Reviews, 10, 371 - 376.

Wagner, U., Gais, S., Haider, H., Verleger, R., \& Born, J. (2004). Sleep inspires insight. Nature, 427, 352-355.

Weizenbaum, J. (1966). Elizaa computer program for the study of natural language communication between man and machine. Communications of the ACM, 9, 36-45.

Xie, L., Kang, H., Xu, Q., Chen, M. J., Liao, Y., Thiyagarajan, M., ODonnell, J., Christensen, D. J., Nicholson, C., Iliff, J. J. et al. (2013). Sleep drives metabolite clearance from the adult brain. science, 342, 373-377. 